\title{
Surgical management of spondylolisthesis overview of literature
}

\author{
Shahzad Sadiq, Adam Meir, S. P. F. Hughes \\ Trauma and Orthopaedics, Charing Cross Hospital, Imperial College School of Medicine, London, W6 8RF
}

\section{Introduction}

Spondylolisthesis (Spondylos=vertebrae; Listhesis= slippage) is forward slippage of one vertebra on another and may be the result of a spondylolysis. The classification by Marchetti and Bartolozzi $^{[1]}$ examines the two broad aetiological groups, namely developmental and acquired (Table 1). This classification has relevance to the natural history, risk of progression and implications of treatment of spondylolisthesis. The Meyerding's classification $^{[2]}$ is used to describe the severity of spondylolisthesis by means of the lateral radiograph to assess the relative anterior translation of the cephalad vertebral body over its distal counterpart.

Spondylolisthesis is easily recognized yet confusion persists over its natural history and preferred treatment. A better understanding of the natural history and the disease pathogenesis is required to allow an evidence based approach to the management of spondylolisthesis.

\section{Natural history, pathology and imaging}

The incidence of spondylolysis is 5-6\% in the general population, however the increased prevalence (up-to 12\%) noted in adolescents with Scheurman's disease, weight lifters, athletes such as football lineman and gymnasts, signifies that mechanical factors may be important in the aetiology of this condition. ${ }^{[3]}$ Several studies suggest a congenital predisposition to spondylolysis, with prevalence's of 27 to $69 \%$ among family members of the affected individuals. ${ }^{[4]}$ Spondylolisthesis is associated with an increased incidence of sacral spina bifida (28\% to $42 \%)$ and congenital deficiency of the sacrum and superior sacral facets. ${ }^{[5]}$ It is thought that, in a dysplastic spine, repetitive or acute traumatic stress on the pars inter-articularis leads to structural failure. ${ }^{[6]}$ The pars defect is thought to be primarily acquired and is seen very rarely in the newborn. ${ }^{[3]}$ The most commonly affected vertebrae are L4 and L5, which are the keystones of the lumbo-sacral spine, providing stability by supporting physiological loads and preventing unnecessary motion.

The long-term follow-up studies have shown that the natural history of this condition is typically benign. ${ }^{[3,7]}$ Progression, neurological deficit and low back pain can occur associated in one study with the Meyerding grade, low sacral index and disc degeneration. $^{[8]}$

With regards to progression, in patients with isthmic spondylolisthesis the incidence of a spondylolysis may be as high as $70 \% .{ }^{[6]}$ The risk of progression from spondylolysis to spondylolisthesis is reported to be small $4 \%-5 \% .{ }^{[2]}$ The risk factors that increase the likelihood of further slippage are younger age, female sex, presence of spina bifida, wedging of the vertebrae, rounding of the anterior sacral dome and hyperlordosis. ${ }^{[9]}$

Spondylolisthesis can be visualized using standard lateral films and forms the basis for Meyerding's Classification. (Figure 1) Oblique radiographs are best for detecting spondylolysis. The Scottie dog sign of Lachapelle is seen on the oblique view as defect in the collar around the dog's neck. CT scan and SPECT scans improve detection rate of spondylolysis. MRI scan is used preoperatively to assess neurological compression and surrounding soft tissue and bony anatomy.

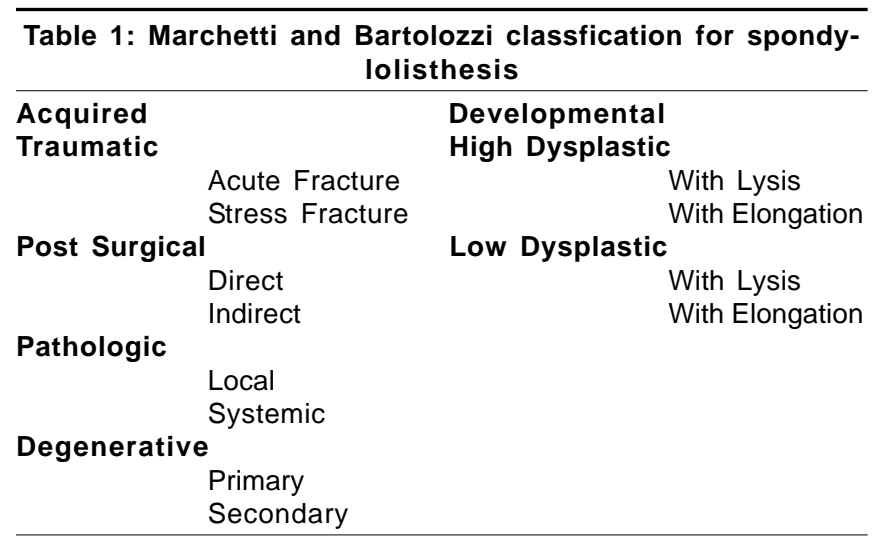




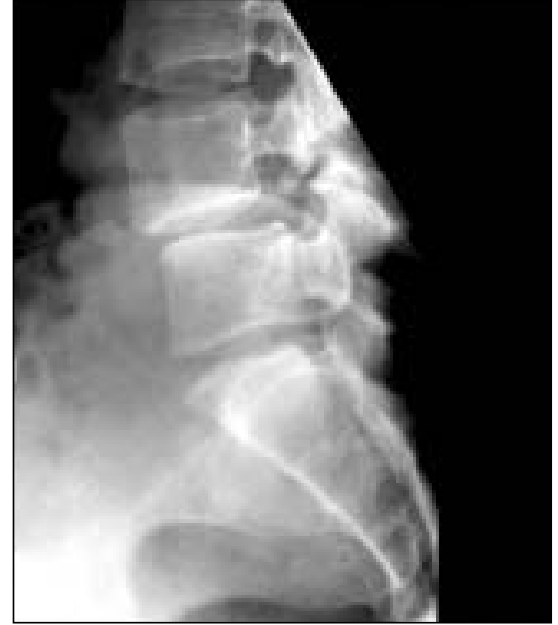

Figure 1: Spondylolysis with Meyerding Grade I spondylolisthesis

\section{Presentation and clinical evaluation}

Children and adolescents usually being asymptomatic but experience an increase in pain during the pubertal growth period. ${ }^{[10]}$ This also occurs in adults with low back pain and sciatica. In the older adult population, stenosis is more of a feature with pain due to degenerative changes more prevalent. In children, postural and gait abnormities due to tight hamstrings are more common. ${ }^{[1]}$

\section{Management}

Management decisions for adults with low-grade acquired spondylolytic spondylolisthesis need to take into account the natural history of the deformity, the severity and duration of symptoms, and patient's co morbidities.

The progression of spondylolisthesis depends heavily on aetiology and age at presentation.

Surgical management depends on symptoms, grade and aetiology, with decision to operate determined mainly by the symptoms and which operation to perform, decided by the later two factors.

We propose that the surgical management could be considered in three broad groups stratified according to age at presentation. (Group, I to III) and further divided into two subgroups using suffix A (low grade 0, I, II) and B (High Grade:- II, III and V).

\section{Group I-A: Children and adolescent with low grade spondylolisthesis}

The asymptomatic child diagnosed incidentally with a spondylolysis or low grade spondylolisthesis requires no specific treatment but should be followed up. Symptomatic children with low back pain should be investigated to establish the diagnosis and exclude other causes of back pain. Non-surgical management primarily consists of activity modification, bracing, physical therapy and intervention in the form of medications or injections. Wiltse and Jackson ${ }^{[6]}$ proposed that activities need not be restricted if a slip is less than 25 percent but if slip is $>25 \%$ than contact sports and activities risking back to be avoided.

Steiner and Michelli ${ }^{[12]}$ assessed bony healing and clinical outcomes in 67 patients with spondylolysis or low grade spondylolisthesis who were treated with a Boston Brace. 12/67 patients showed evidence of bone healing at four months and $78 \%$ were reported to have good results.

Surgical treatment may be considered in spondylosis and grade I slips in those patients who fail to respond to prolonged conservative management (Figures 1-3). Indications include progressive slip, intractable back and leg pain and neurological deterioration.

The role of direct repair is controversial. Where the pars defect is combined with minimal slip, it is an attractive alternative, as popularized by Buck ${ }^{[13]}$ and then by Bradford and Iza. ${ }^{[14]}$ However, this is suitable only in a selected group where no instability or nerve compression is present. Dai et $\mathrm{al}^{[15]}$ demonstrated union rate of $94 \%$ of pars defect and a satisfactory clinical result in patients with lumbar isthmic spondylolisthesis and mild spondylolisthesis in 46 patients. These authors found that use of MRI was of great assistance in developing the protocol choice of whether to include facet joint fusion.

In a prospective randomized study Moller and Hedlund ${ }^{[16]}$ compared the outcome after postero-lateral fusion in patients with adult isthmic spondylolisthesis or managed with an exercise program. The functional outcome, as assessed by the Disability Rating Index and the amount of pain reduction, was better in the surgically treated group than in the exercise group at both the one and two-year follow- up assessments.

\section{Group I- B: Children and adolescent with high grade slips}

Surgical treatment is indicated for slips greater than $50 \%$ in both symptomatic or asymptomatic patients. Neurological symptoms despite non-operative management, progression and mechanical deterioration generally require some sort of operative intervention.

The standard surgical treatment in this age is group is bilateral inter-transverse process fusion in situ (Figures 2a and b). In slippages of less than $50 \%$ this involves a single level fusion from L5-S1, with the fusion extending to L4 with more severe slippages. This approach has shown medium to good results with follow up to 14 years. ${ }^{[17-19]}$ Isolated decompression without fusion is contraindicated in children. Wiltse and Rothman ${ }^{[20]}$ suggested that decompression is rarely required in children, stating that neurologic deficits improve after fusion.

There is not enough in the literature to recommend the routine use of instrumentation for children as the results of isolated fusion are good and fusion is achieved relatively quickly in short time. There are some reports however of the use of transpedicular fixation to improve reduction and stabilize the spine hence avoiding postoperative splintage.

\section{Group II-A: Adults with low grade spondylolisthesis}

If non-operative treatment fails the mainstay of surgical treatment for adult patients with low-grade acquired spondylolytic spondylolisthesis is fusion, with or without decompression. Direct 
pars repair in adult patients or decompression alone (i.e. using Gill's procedure) are operations that are not typically recommended in adults. ${ }^{[21]}$

The fusion techniques available for this deformity can be divided into those which achieve posterior column stability (i.e, posterolateral intertransverse fusion $[\mathrm{PLF}]$ ) or those that achieve anterior column stability (i.e., anterior lumbar interbody fusion [ALIF]) or when both columns are stabilized.

\section{Group II-B: Adults with high grade spondylolisthesis"}

The ideal treatment for symptomatic Meyerding Grade III-IV spondylolisthesis remains controversial, with a variety of techniques being described. ${ }^{[22,23]}$

The radiographic appearance in these patients may be alarming. However it is worth noting that some adults with high grade spondylolisthesis have little or no pain. Other patients who present with low back pain should be evaluated carefully to exclude a disc

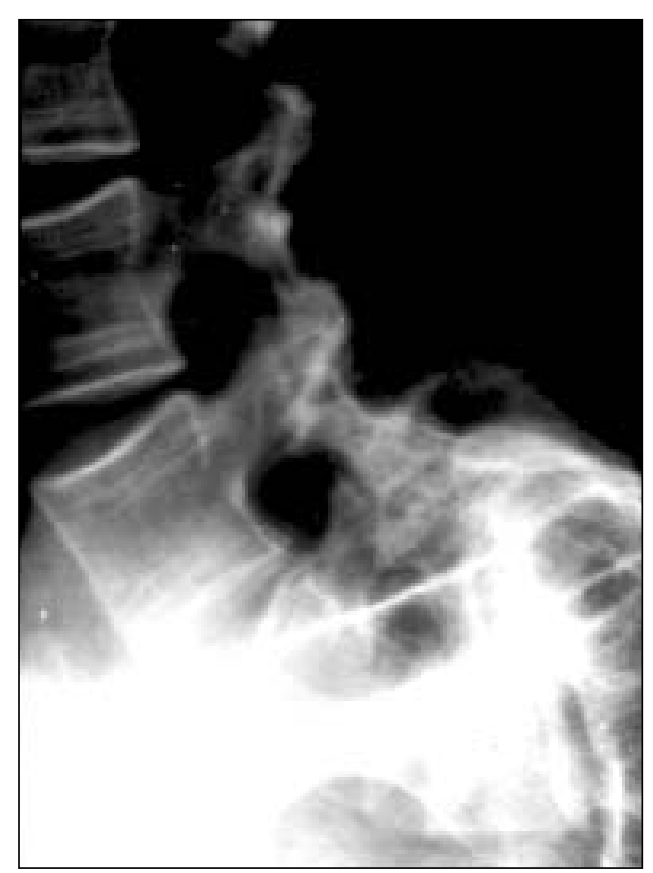

Figure 2A: Group I patient treated with in-situ fusion

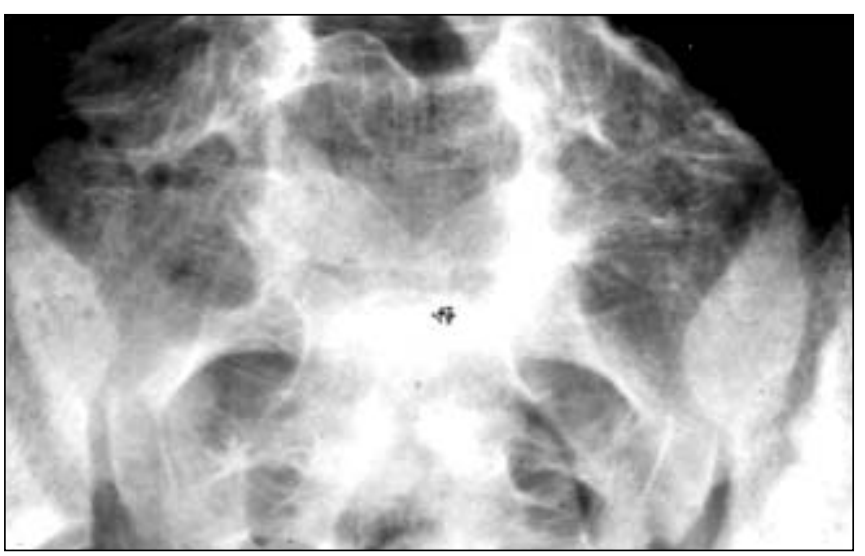

Figure 2B: Group I patient treated with in-situ fusion prolapse more proximally. Physiotherapy should be provided, but it is prudent to identify adults who have high amount of bony dysplasia. It is unusual for an acquired type of spondylolisthesis to progress to a high grade slip. In view of this and the definite risk of complications especially if any sort of reduction is attempted, very careful consideration needs to be made regarding the aim of any intervention and a risk benefit analysis performed for each individual patient.

If low back pain is present and thought to be due to instability, there is obviously a wide variety of procedures that can be performed. The main stay of operative intervention however has been fusion in-situ with or without instrumentation. Posterior in situ arthrodesis without laminectomy has shown to produce good pain relief with no pseudo-arthroses. ${ }^{[22]}$ However, despite the clinical successes, pseudarthrosis rates have been reported up to $40 \%{ }^{[23]}$ In addition, due to the severe stresses imposed, solid fusion masses without instrumentation (Figure 3) can bend allowing the spondylolisthesis to progress. To improve the fusion rates, most proponents of in situ arthrodesis now advise the addition of instrumentation with fixation recommended from L4 to $\mathrm{S} 1$, including L5 pedicle screws if possible. This is now the procedure of choice for high-grade spondylolisthesis in the adult, particularly for revision cases with failed arthrodesis.

In terms of overall spinal mechanies, the end result of adults with a high-grade spondylolisthesis, who have remained minimally symptomatic during their adolescent and young adult growth periods, is often a sagitally balanced deformity. Leading to the conclusion that symptoms must drive treatment rather than radiographic appearance.

Attempts at reduction are controversial as is clear that complications can be severe and extensive discussion with the patient needs occur before surgery is planned. With regards to

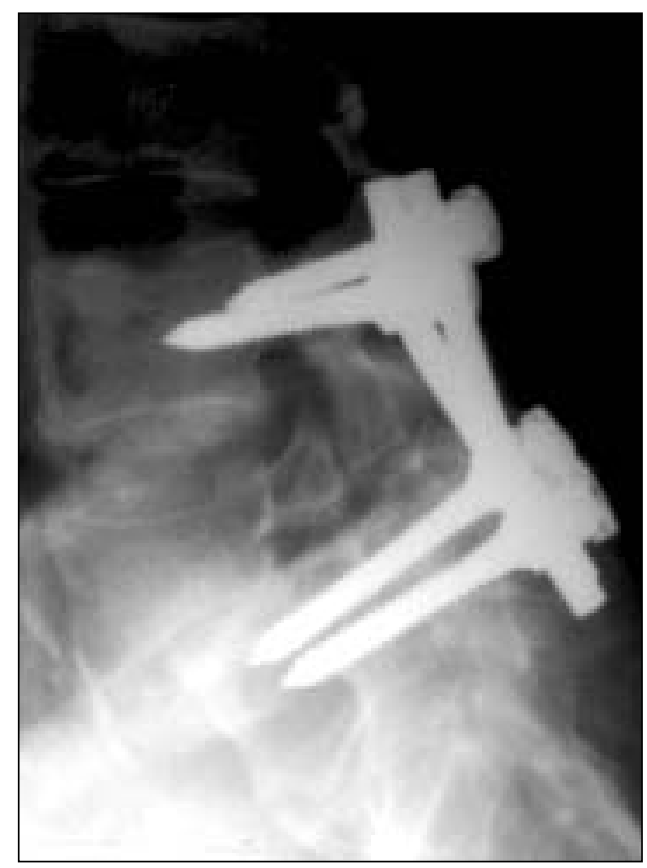

Figure 3: Group II B patient treated with in situ pedicle screw fixation 
decision making and risk assessment, it is very important to establish the nature (developmental or acquired) of the slip. Many high grade slips are iatrogenic after a destabilizing procedure of the underlying soft tissues including the disc, ligaments and facet joints and are akin to post-traumatic kyphosis. Reduction of such slips are less hazardous than developmental types of slips. Developmental slips are never aligned normally, thus all tissue including neurologic tissue must stretch after reduction. Infact this is similar to the lengthening of congenitally shortened leg versus a traumatically shortened leg. ${ }^{[23]}$

Reduction manoeuvers may be considered when there is evidence of segmental instability or when significant sagittal imbalance is present. Reduction typically needs only to be partial to allow a reversal of the lumbosacral kyphosis. It has been recognized that partial reduction of the slip angle and not the percentage of translation is more important in restoring sagittal balance.

Bradford and Boachie-Adjei ${ }^{[24]}$ reported a series of 19 patients including 6 adults who underwent partial reduction after posterolateral arthrodesis. Following posterior laminectomy, the patients underwent halo-skeletal traction and a staged anterior interbody arthrodesis. Neurological injury occurred in 3 of the patients, including a cauda equine syndrome. Reduction of the high-grade spondylolisthesis improved the sagittal profile of 17 of the 19 patients. The authors concluded that reduction had several advantages namely improved biomechanical orientation to facilitate arthrodesis, neurological decompression, correction of the lumbosacral kyphosis, and restoration of sagittal alignment. They also felt that the patient's abnormal posture and gait were a major disability and that correction of the deformity via partial reduction was essential.

There have been a variety of different methods of fusion with or without instrumentation noted in the literature. These are: Instrumented posterolateral fusion, ${ }^{[25,26]}$ anterior lumbar interbody fusion (ALIF), ${ }^{[27]}$ transforaminal interbody fusion (TLIF), ${ }^{[28]}$ Instrumented PLIF. ${ }^{[29]}$ It seems clear that in terms of clinical outcomes there is no evidence that any technique is superior. In addition, the length of follow up is variable, making comparisons difficult. It is however important to note that posterior fusions with or without pedicle screw fixation are prone to loss of reduction. This is not the case with anterior fusion. ${ }^{[27]}$ Ishihara achieved low pain scores and $83 \%$ union with 13.3 years follow up, although, adjacent disc degeneration may become a significant variable long term. It will be important to determine the importance of each of these various factors if any rational approach to the different techniques can be determined in the future.

In general, therefore techniques that achieve posterior column stability are the most straightforward and historically the most popular means of performing fusion. ${ }^{[29,30]}$ Posterior surgical techniques allow for direct decompression of the neural elements, deformity correction if desired, and the achievement of stability with pedicle screw instrumentation. The posterior exposure and obligatory stripping of paraspinal musculature, has been postulated as contributing to ongoing low back pain and has been described as "fusion disease." In addition, as stated previously, the PLF does not biomechanically address the anterior column and is recognized to have a less optimal fusion rate. Even in the face of a solidly healed PLF, persistent strain anteriorly may cause ongoing discogenic low back pain. This was suggested by Barrick et $\mathrm{al}^{[31]}$ in their report of patients who continued to suffer low back pain despite solid PLF, and who then went on to be successfully treated with ALIF.

Along the same lines, L'Heureux et $\mathrm{al}^{[32]}$ and La Rosa et $\mathrm{al}^{[33]}$ have reported that, in adult low-grade spondylolisthesis patients treated with PLF, the correction in slip angle that was achieved intraoperatively was lost over time as the result of progressive disc space degeneration anteriorly. This again suggests

that the rigidity of a solid PLF does not necessarily protect the disc from the

biomechanical loading that can induce further degenerative changes.

\section{Group III-A and B}

Adults older than 40 with low Grade spondylolysis and spondylolisthesis

Degenerative spondylolisthesis rarely exceeds Grade I and II and is common in elderly females usually affecting the lumbar 45 segments. In these patients the initial treatment is non-operative. However, persistent or recurrent back or leg pain or neurogenic claudication with significant reduction in quality of life and failure of conservative trial of treatment, along with progressive neurology or bladder or bowel symptoms, are all indications for surgery.

The mainstay of surgical treatment is decompression and the goal here is to relieve radicular symptoms and neurogenic claudication. Fusion is required more often where instablilty is predominant and primary complaints are long standing backache due to degenerated discs and facetjoints. Instrumentation may improve fixation results but is also associated with a higher complication rate.

There has long been controversy whether isolated decompression is ever indicated. Lombardi et $\mathrm{al}^{[34]}$ reviewed the results of decompression alone in 47 patients with a follow up of 2-7 years. The results were much better for partial facetectomy and isolated fusion compared with complete facetectomy.

Herkowitz and Kurz ${ }^{[35]}$ in a prospective study compared the results of decompression alone and decompression with fusion in 50 patients. The fusion group showed better outcomes and lesser progression of the slip, at mean follow up of three years. Other studies have also shown better results with fusion and are marginally better with instrumented fusion. ${ }^{[36,37]}$ The Cochrane database was against fusion while Swedish Spine Study Group concluded in favour of fusions, consistent findings being that instrumentation in posterior fusions increased the fusion rate. ${ }^{[23]}$

Anterior fusion posterior decompression has also been found to achieve good results compared with, presumably stabilising effectively and achieving indirect decompression by improving disc height. ${ }^{[36,37]}$ Satomi et al, ${ }^{[38]}$ reported $77 \%$ good results in 27 patients with ALIF as compared to $56 \%$ good results with posterior 
decompression in 14 patients.

Adjuncts to the biology of fusion such as Bone Morphogenic Proteins and other Bone substitutes have been used. Johnsson et $\mathrm{al}^{[39]}$ compared the results of fusion using BMP and without BMP and found no difference in fusion rate. Boden et $\mathrm{al}^{[40]}$ however in their study showed improved fusion rates and better clinical results with use of Bone Morphogenic Protein and bone substitute hydroxyappatite and tricalcium phosphate. Dynamic stabilization devices include spinous process distraction devices, ligaments across the pedicle screws and semi-rigid plastic or metallic devices across the pedicle screws. ${ }^{[11]}$ There is no clear consensus over the results or use of these devices in surgery for spondylolisthesis hence they cannot be recommended at this stage. They may however in the future offer an alternative to solid fusion particularly in the young age group where long term adjacent disc degeneration may be problematic.

\section{Maintaining balance in degenerative spondylolisthesis}

Bednar ${ }^{[42]}$ reported the outcome of posterior instrumented fusion for degenerative spondylolisthesis with reduction of the listhesis using a pedicle screw. Though clinical results were good but loss of disc height was evident in radiological follow up. This study advocates the use of inter-body graft if reduction is attempted.

\section{Conclusion}

Spondylolysis and spondylolisthesis of developmental in origin are found in $5 \%$ of children and $7 \%$ of the adults. Most patients have no symptoms and their condition remains stable, those with symptoms and higher grade slips require further investigation. Surgical management should take into account the natural history of the disease, the age at presentation and the severity of the slips. Children respond well to bracing and bony fusion using the Wiltse approach. The management in adults should focus on the symptoms related to instability, disc degeneration and spinal alignment. Developmental spondylolisthesis should be differentiated from acquired spondylolisthesis that usually presents later in life leading to spinal stenosis, instability and root canal stenosis. Careful patient selection and clinical patterns will determine the choice of surgical procedure from simple decompression to fusion. Instrumented fusion improves the fusion rate however complications from insertions of screws also increase. Dynamic instrumented fusion concept is appealing but long-term results are being evaluated.

\section{References}

1. Marchetti PG, Bartolozzi P. Classification of spondylolisthesis as a guideline for treatment. In: Bridwell KH, Dewald RL, Hammerberg KW et al, editors. Textbook of Spinal Surgery, 2nd edn. Philadelphia: Lippincott-Raven; 1997. p. 1211-54. 2. Meyerding HW. Spondylolisthesis. Surg Gynecol Obstet 1932;54:371.
3. Fredrickson BE, Baker D, McHolick W.J, Yuan HA, Lubicky JP. The natural history of spondylolysis and spondylolisthesis. J Bone Joint Surg Am 1984;66:699-707.

4. Albanese M, Pizzutillo PD. Family Study of spondylolysis and spondylolisthesis. J Paediatr Orthop 1982;2:496-9.

5. Wynne-Davies R, Scott JH. Inheritance and spondylolisthesis: A radiographic family survey. J Bone and J Surg Br 1979;61B:301-5.

6. Wiltse LL, Jackson D. Treatment of spondylolisthesis and spondylolysis in children. Clin Orthop 1976;117;92.

7. Saraste H. Long term clinical and radiological follow-up of spondylolysis and spondylolisthesis. J Paediat Orthop 1987;7:631-8.

8. Hammerberg KW. New concepts on the pathogenesis and classification of Spondylolisthesis. Spine 2005;30;S4-11.

9. Lonstein JE. Spondylolisthesis in children: cause, natural history, and management. Spine $1999 ; 24: 2640-52$.

10. Lafond G. Surgical treatment of spondylolisthesis. Clin Orthop 1962;22:175.

11. Moller H, Sundin A, Hedlund R. Symptoms, signs, and functional disability in adult spondylolisthesis. Spine 2000;25:683-9 results in 72 consecutive cases. Acta Neurochir (Wien) 1991;110:154-9.

12. Steiner ME, Micheli IJ. Treatment of symptomatic spondylolysis and spondylolisthesis with the modified Boston Brace. Spine 1985;10:937-45.

13. Buck JE. Direct repair of the defect in spondylolisthesis. J bone and Joint Surg Br 1970;52:432-7.

14. Bradford DS, Iza J. Repair of the defect in spondylolysis or minimal degrees of spondylolisthesis by segmental wire fixation and bone grafting. Spine 1985;10:6739.

15. Dai LY, Jia LS, Yuan W, Ni B, Zhu HB. Direct repair of defect in lumbar spondylolisthesis and mild isthmic spondylolisthesis by bone grafting, with or without facet joint fusion. Eur Spine J 2001;10:78-83.

16. Moller H, Hedlund $\mathrm{R}$. Surgery versus conservative management in adult isthmi spondylolisthesis:a prospective randomized study: part 1 . Spine 2000;25:17115 .

17. Johnson .JR, Kirwan EO. The long-term results of fusion in situ for severe spondylolisthesis. J Bone Joint Surg Br 1983;65:43-6

18. Seitsalo S, Osterman K, Hyvarinen H, Schlenka D, Possa M. Severe spondylolisthesis in children and adolescents. A long term review of fusion in situ. J bone Joint Surg Br 1990;72:259-65.

19. Frennered AK, Danielsen BL, Nachemson AL, Nordwall AB. Midterm follow up of patients fused in situ for spondylolisthesis. Spine 1991;16:409-16.

20. Wiltse, Rothman LG. Spondylolisthesis: Classfication, diagnosis, and natural history Semin Spine Surg 1989;1:78-94.

21. Gill G, Manning J, White H. Surgical Treatment of spondylolisthesis without fusion. Excision of the loose lamina with decompression of nerve roots. J Bone and Joint Surg Am. 1955;37:493-520.

22. Peek RD, Wiltze LL, Reynolds JB, Thomas JC, Guyer DW, Widell EH. In situ arthrodesis without decompression for Grade III and IV isthmic spondylolisthesis in adults who have severe sciatica. J Bone Joint Surg Am 1989;71:62-8.

23. Dewald C.J, Vartabedian JE, Rodts MF, Hammerberg KW. Evaluation and management of high grade spondylolisthesis in adults. Spine 2005;30:49-59.

24. Bradford DS, Boachie-Adjei O. Treatment of severe spondylolisthesis by anterior and posterior reduction and stabilization:a long-term follow-up study. J Bone Joint Surg Am 1990;72:1060-6.

25. Moller H, Hedlund R. Instrumented and non-instrumented posterolateral fusion in adult spondylolisthesis:a prospective randomized study: part 2. Spine $2000 ; 25: 1716-21$

26. Suk SI, Lee CK, Kim WJ, Lee JH, Cho KJ, Kim HG. Adding posterior lumbar interbody fusion to pedicle serew fixation and posterolateral fusion after decompression in spondylolytic spondylolisthesis. Spine 1997:22:210-9.

27. Ishihara H, Osada R, Kanamori M, Kawaguchi Y, Ohmori K, Kimura T, et al. Minimum 10 -year follow-up study of anterior lumbar interbody fusion for isthmic spondylolisthesis. J Spinal Disord 2001;14:91-9.

28. Rosenberg WS, Mummaneni PV. Transforaminal lumbar interbody fusion: Technqiue, complications, and early results. Neurosurgery 2001;48:569-74.

29. Molinari RW, Gerlinger TA. Functional outcomes of instrumented PLIF in active duty US army soldiers; a comparison with non operative management. Spine J 2001;1:215-24

30. Csecsei GI, Klekner AP, Dobai J, Lajgut A, Sikula J. Posterior interbody fusion using laminectomy bone and transpedicular screw fixation in the treatment of lumbar spondylolisthesis. Surg Neurol 2000;53:2-6.

31. Barrick WT, Schofferman JA, Reynolds JB, Goldthwaite ND, McKeehen M, Keaney D, et al. Anterior Lumbar Fusion improves discogenic pain at levels of prior posterolateral fusion. Spine 2000;25:853-7.

32. L Heureux EA Jr; Perra JH, Pinto MR, Smith MD, Denis F, Lonstein JE. Functional outcome analysis including preoperative and postoperative SF36 for surgically treated adult isthimic spondylolisthesis. Spine 2003;28:1269-974.

33. L Rosa G, Conti A, Cacciola F, Cardali S, La Torre D, Gambadauro NM, et al. Pedicle screw fixation for isthmic spondylolisthesis: does posterior lumbar interbody fusion improve outcome over posterolateral fusion? J Neurosurg 
2003;99:143-50

34. Lombardi JS, Wiltse LL, Reynolds J, Widell EH, Spencer C $3^{\text {rd }}$. Treatment of degerative spondylolisthesis. Spine 1985;10:821-7.

35. Herkowitz HN, Abraham D.J, Albert T.J. Management of degenerative dise disease above an L5-S1 segment requiring arthrodesis. Spine 1999;24:1268-970.

36. Mardjetko SM, Connolly PJ, Shott S. Degenerative Lumbar Spondylolisthesis: metaanalysis of literature 1970-1993. Spine 1994;19:2256-64.

37. Yaun HA Garfin R, Dickman CA, Mardjetko SM. A historical cohort of pedicle screw fixation in thoracic, lumbar and sacral spinal fusions. Spine 1994;19:2279-96.

38. Satomi K, Hirabayashi K, Toyama Y, Fujimura Y. A clinical study of degenerative spondylolisthesis: radiographic analysis and choice of treatment. Spine 1992:17;1329-36.

39. Johnssaon R, Stromqvist B, Aspenberg P. Randomized radiosterometric study comparing osteogenic protein-1 (BMP-7) and autograft bone in human noninstrumented posterolateral lumbar fusion: 2002 Volvo Award in clinical studies. Spine 2002;27:2654-61.

40. Boden SD, Kang J, Sandhu H, Heller JG. Use of recombinant human bone morphogenic protein-2 to achieve posterolateral lumbar spine fusion in humans: a prospective, randomized clinical pilot trial. 2002 volvo award in Clinical studies. Spine $2002 ; 27: 2662-73$.

41. Hammerberg KW. Spondylolysis and spondylolisthesis. In: Dewald RL, ed Spinal Deformities. The Comprehensive Text, $1^{\text {st }}$ Edn. New York: Theime; 2003. p. $787-$ 801.

42. Bednar DA. Surgical management of lumbar degenerative spinal stenosis with spondylolisthesis via posterior reduction with minimal laminectomy. J Spinal Disord Tech 2002;15;105-9. 